\title{
Talking Gloves: Sign Language to Speech Conversion for Deaf and Mute Person
}

\author{
Sarvottam N Katti, Sourabh S Sirsi, Vandana Prakash, Vinith Raj B.U, Vandana Jha \\ Department of Computer Science and Engineering, KSSEM, Bangalore
}

\begin{abstract}
Communication is the act of sharing information to produce greater understanding. It is both, an art and science. Like us humans, animals also have its own way of conveying information. We have evolved from one language and have been able to converse in various languages. IoT based Smart Gloves is an innovative system which will help the dumb interact with the normal people. This system uses a set of sensors that are attached to each finger on both hands. Each motion made and the bending of the fingers has its own separate meaning. The gloves read the motions and the orientation of the sensors. The basic task of this work is to develop a portable, reliable and an inexpensive prototype system.
\end{abstract}

Keywords - Accelerometers, Artificial Neural Networks, Flex Sensor, Sign Language.

\section{INTRODUCTION}

Sign language is used by the speech impaired people to converse with normal people. Conveying their messages to people who have no knowledge of the sign language causes great difficulty and leads to a communication gap. Normally, the speech impaired conveys their message by making hand movements along with the fingers. Each hand and finger movement has its own specific meaning. To bridge this gap and help people communicate easily with the others, Talking Gloves are introduced [1][4].
Talking Gloves is a system which consists of flex sensors on the gloves that convert the sign language into an audio output using Arduino. The audio message is only played if the sensors' value matches the gesture made. The gloves read the motion gestures using Flex Sensors sewed within. Once the data is read, Arduino processes the data and then allows the audio output.

If the gloves are not in use, the device has the capability to change into power saving mode to reinforce the battery life. The basic task of this work is to develop a reliable, portable and an economical prototype system which can be affordable to the middle class as well as easily available in the market.

\section{A. Motivation}

The main motivation behind developing this system is to design a system which can recognize hand movements and gestures and facilates a medium of communication to the people who cannot communicate by talking. These hand movements should be recognized and should be converted into words and provide natural way of communication just like talking. Giving 45 instructions to the Gloves and converting those instructions in words are easy but giving the functionality of natural communication similar to talking is very difficult. Some other objectives for developing the system are as follows:

\section{Independence from the remote unit}


The talking gloves should be operated by battery power and the information transmitted from this gloves to the unit which is kept remotely from this portable system should be wireless utilizing a nonstandard convention to downsize the consumption of power. This prototype system should be comfortable and lightweight, which can be wore easily because it is developed for the people who are just dumb or for the patients who may have some problems in movement and their muscles might be weak. Anyways as it is a wearable device, its comfortability and weight matters a lot.

\section{Economical}

The device or the prototype should be economical to the pocket of the middle class then only it will be used frequently in the hospitals, homes and rehabilitation centers. It's low cost will make it affordable by the masses.

\section{Positioning of Sensors}

The sensors should be attached to the gloves in such a way that it can collect the information by the movement of fingers and hand. It should able to record the pressure applied by each finger joints and their movements. By considering these needs, the sensors should be sewed to the gloves at the top of every finger as shown in the fig. 1 .

\section{B. Organization}

The paper is introduced in section I with the motivation behind its development. Section II showcases the existing work and systems available in the market with similar functionality. The components used in developing this system and their working are given in section III. The development methodology and steps involved in its implementation is provided in section IV. The paper is concluded in section $\mathrm{V}$.

\section{LITERATURE REVIEW}

The existing system for recognizing the hand gesture for the Indian sign language requires the use of Cam Shift, HSV model and the recognizing the gestures through genetic algorithm [1][2].

Another system for recognizing Indian sign gestures was proposed in which the gestures were perceived through left and right scan of 7-bit orientation and generation process. Every gesture was captured by a method specific state change which was dependent upon normal human verbalization.

The static emotions were perceived utilizing picture minutes while dynamic motions were perceived by dissecting their moving directions.

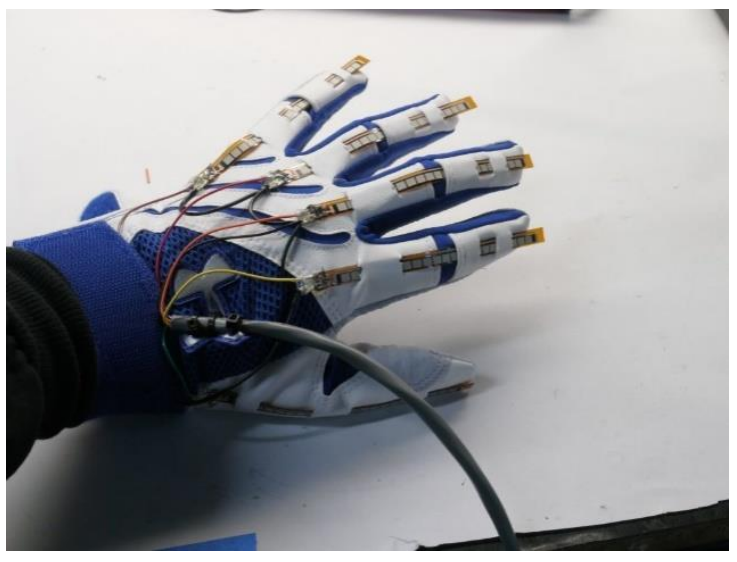

Fig. 1: Design Architecture of Talking Gloves

\section{WORKING}

\section{System Development Components}

\section{Sensors}

A sensor can be a subsystem, device, module or a machine whose purpose is to sense changes in its setting and send the data to alternative electronic devices, often times a pc processor.

A flex sensor or a bend sensor can be a sensor that measures the number of bending or deflection. Usually, the sensing 
element or device is stuck to the surface and also the resistance of the sensor element is varied by bending the sensor. The resistance is directly proportional to the number of bend. Hence, it can be used as a Goniometer. A flex sensor is widely employed in the areas of analysis from pc interfaces, in the research domain, rehabilitation, security systems and even music interfaces [1][2].

\section{Arduino Mega Board}

ATmega2560 has been utilized along with Raspberry $\mathrm{Pi}$ as the processor for this prototype. A Gyro and Flex sensor is utilized to detect the movement and furthermore the twist of the fingers. A display is utilized to show the message. Arduino Mega board is built on ATmega 2560. Total 100 pins are there on Arduino Mega board. Among these pins, analog pins are 16 and digital pins are 54. It also has 4MB Electronically Erasable Programmable Read only Memory (EEPROM) and 256 Kbytes flash memory. It is used at $16 \mathrm{MHz}$ frequency. ATmega 2560 is the 8-bit micro-controller. The default bit pace of information transmission is 9600. It's furthermore empowered in Universal Synchronous Asynchronous Receiver Transmitter (USART), Serial Peripheral Interface (SPI) and Inter-integrated Circuit (I2C) [2][4].

Arduino Mega has a partner onboard transformer, So, it's proficient to require partner input voltage from $6 \mathrm{~V}$ to $20 \mathrm{~V}$. Anyway it's directed to control partner input voltage from $7 \mathrm{~V}$ to $12 \mathrm{~V}$ and highest input current $500 \mathrm{~V}$. Pin yield voltage $5 \mathrm{~V}$ and yield current is $20 \mathrm{~mA}$. It has furthermore inbuilt power supply which can offer $3.3 \mathrm{~V}$ and $5 \mathrm{~V}$. The $3.3 \mathrm{~V}$ pin has $50 \mathrm{~mA}$ current. Arduino Mega board is shown in the fig. 2 .

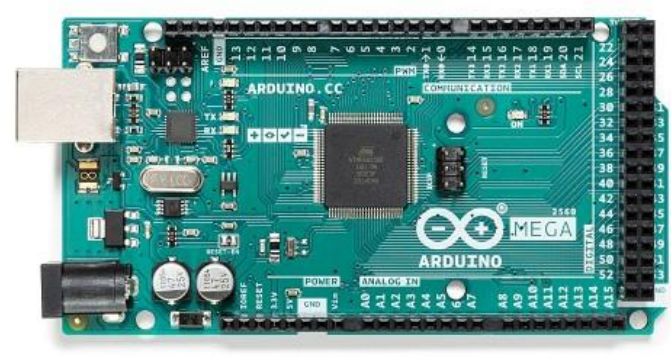

Fig. 2: Arduino Mega Board

\section{MPU6050}

MPU6050 is utilized for detecting the movement of the hand. MPU 6050 is an integrated 6-axis movement detection gadget. This can be joined with a 3-axis accelerometer, a 3-axis gyroscope, a 3-axis compass and with a Digital Motion processor (DMP). For digitizing the output of gyroscope that gives access to the program at full scale differ of $\pm 250, \pm 500$, \pm 1000 , and $\pm 2000^{\circ} / \mathrm{sec}$ (DPS), it has three analog to digital converters (ADC).

\section{Raspberry Pi}

The Raspberry Pi as shown in fig. 3 , is made in three board configurations through licensed manufacturing deals with Newark element14 (Premier Farnell), RS Components and Egoman. The Raspberry Pi incorporates a Broadcom BCM2835 framework on a chip (SoC), which has an ARM1176JZF-S 700 rate processor, Video Core IV GPU, and was initially dispatched with 256 megabytes of RAM, later updated (Model B and Model B+) to 512 MB. It doesn't typify an inbuilt hard disk or strong state drive, anyway it utilizes an SD card for booting and extended stockpiling, with the Model B+ utilizing a MicroSD [1][3][4].

The Broadcom SoC utilized in the Raspberry Pi is similar to a chip used in an old Smartphone (Android or iPhone). Although operational at $700 \mathrm{MHz}$ as a matter of course, the Raspberry Pi gives a genuine world exhibition generally equal 
to 0.041 GFLOPS. The illustration capacities of the Raspberry Pi are generally equal to the degree of execution of the Xbox of 2001.

A Bluetooth module HC-06 goes about as an imparting between hand gloves and an android phone. It utilizes radiofrequency radio wave. Bluetooth works in conviction band at a couple of 4GHz.HC-06 follows the Bluetooth V2.0 convention. It has the operational voltage of $3.3 \mathrm{~V}$ and current $40 \mathrm{~mA}$.

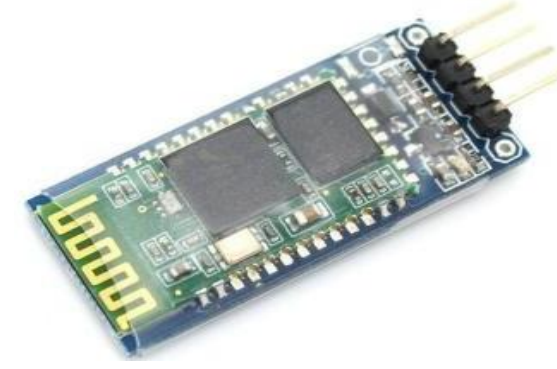

Fig. 3: Raspberry Pi

\section{Programming Language Selection}

$\mathrm{C}$ is used as the programming language. $\mathrm{C}$ is a fundamental procedural language. It has been intended to be accumulated utilizing a relatively simple compiler to deliver low-level access to memory and language builds that map with effectiveness to machine bearings, all with insignificant runtime support. Regardless of its low-level capabilities, the language was intended to promote crossplatform programming [1][3].

A norms agreeable PC program composed in light of versatility will be ordered for a huge sort of pc stages and usable frameworks with few changes to its ASCII text record. The language is offered on various platforms, from installed microcontrollers to supercomputers.

C has capabilities for structured programming and permits lexical variable degree and recursion. Its static kind framework forestalls incidental operations. In $\mathrm{C}$, all conceivable code is contained inside subroutines (otherwise called "functions", not rigorously inside the feeling of functional programming).

A few things that $\mathrm{C}$ is regularly utilized for are:

- Web Development

- Game Programming

- Desktop GUIs

- Scientific Programming

- Network Programming

\section{METHODOLOGY}

Hardware Assembly Six flex sensors are connected on the thumb, index, middle, ring, little fingers and therefore the palm of a glove so as to live the bend of the fingers and therefore the clench of the hand then measuring instrument and therefore the rotating mechanism was placed on the rear of the hand so as to work out the position and movement of the hand on area. Sensors were interfaced with Arduino mega 2560 [1] [4].

\section{Dataset Collection}

Due to inaccessibility of the datasets for coaching the machine with our sensors knowledge the dataset for coaching the model was created wherever data at the interface for every gesture representing the alphabets and a few frequent used words were collected and saved in comma-separated values (CSV) file format.

\section{Data Processing:}

All the information collected with numerous options as per the gesture were categorized with their various alphabet or word as their target value, and also the final collected information was randomized so as to scale back the variance and to confirm that the model remains general and not overfit.

\section{Dataset Description:}

A total of 15050 data (after all preprocessing steps) taken at completely different times, conditions, and settings together with the $\{$ various $\}$ positions of 
the hand on area for an equivalent class similarly as well as various other categories [2][3].

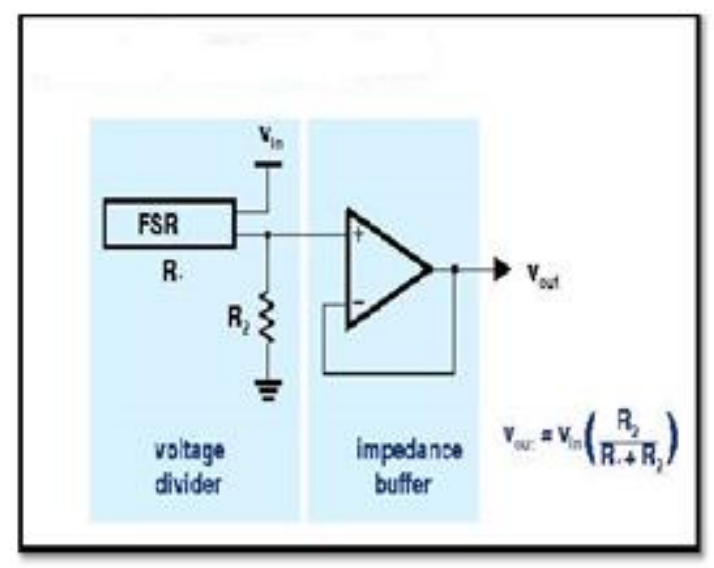

Fig. 4: Basic Flex Sensor Circuit

The fig. 4 shows the fundamental circuit diagram of the flex device. Utilizing flex device, we tend to live the modification in resistance motion Sensors (Flex Sensor) - The flex devices area unit the sensors that modification in resistance relying upon the quantity of bend on the sensor. They convert the modification in bend to electric resistance. They are unidirectional and bidirectional, present in the market in the form of skinny strip kind [1][3].

The important distinction between an unidirectional flex device and a bidirectional flex device is that, because the unidirectional flex device is bent, the resistance will increase, whereas once a bidirectional flex device is bent, the resistance decreases.

\section{CONCLUSION}

Deaf and dumb individuals are dependent on sign language interpreters for communication. However, they cannot rely upon interpreters every day in life principally thanks to the high prices and also the problem in finding and hiring qualified interpreters.

The impaired individuals use their normal sign language but that is not simple to understand for the normal people. This technique converts the gestures into voice that is definitely perceivable by the individuals. The sign language is translated into voice note, to facilitate the impaired people to convey their messages to others.

In today's time when IoT is finding its space in various sectors, the work and research done in this field inspire us to find various smart solutions to the problems existing in our society.

\section{REFERENCES}

[1] Bui, TT Quyen, and Keum-Shik Hong, "Evaluating a color-based active basis model for object recognition," Computer Vision and Image Understanding, vol. 116, no. 11, pp.1111-1120, 2012.

[2] Oudah, Munir, Ali Al-Naji, and Javaan Chahl, "Hand gesture recognition based on computer vision: a review of techniques," Journal of Imaging, vol. 6, no. 8, pp.73, 2020.

[3] Dipietro, Laura, Angelo M. Sabatini, and Paolo Dario, "A survey of glovebased systems and their applications," IEEE transactions on systems, man, and cybernetics, part c (applications and reviews), vol. 38, no. 4, pp. 461-482, 2008.

[4] Wang, Chunli, Wen Gao, and Shiguang Shan, "An approach based on phonemes to large vocabulary Chinese sign language recognition," In Proceedings of Fifth IEEE International Conference on Automatic Face Gesture Recognition, pp. 393-398, 2020. 